\title{
Efficient nanoarray catalyze carbon dioxide conversion
}

\author{
Qineng $\mathrm{Xu}^{1, *}$ \\ ${ }^{1}$ Northeastern University, College of Sciences Department, NO. 3-11, Wenhua Road, Heping District, Shenyang, China
}

\begin{abstract}
With the rapid development of industrialization, the increasing production and emission of carbon dioxide leads to strong greenhouse effect and global warming. According to survey, the average temperature has been continuously increased and the rate of increasement may accelerate. Among the solutions people proposed, the utilization of carbon dioxide conversion is widely favored. At the same time, the emerging nanomaterials have made a breakthrough in the past decade. Due to its characteristic properties, nanomaterials have an advantage over traditional catalyst. Under above background, this paper reviews carbon dioxide conversion and nanocatalyst. Meanwhile, we also propose to apply nanoarray in carbon dioxide conversion, aim to make contribution to the utilization of carbon dioxide and slow down global warming.
\end{abstract}

\section{Background}

Carbon is one of the most important elements that makes up lives on earth. Carbon dioxide, as the most widely existing carbon source in the atmosphere, can be used as the basic component of the natural material circulation and participate in the "carbon-organic carbon-carbonate" circulation which represents the relationship between the atmosphere, terrestrial biosphere, pedosphere and oceansphere. In recent years, with the continuous development of industry and human society, the energy consumption is much more than before, which leads to the exploitation of natural resources. Among them, the intensive depletion of fossil fuels has resulted in a swift rise of the concentration of carbon dioxide in the atmosphere.[1,2]Meanwhile, the destruction of vast areas of forest and grasslands gave rises to a sharp decline of vegetative cover on the Earth's surface which decrease the photosynthesis of plants indirectly caused the higher concentration of carbon dioxide in the atmosphere. As we all know, the increase of carbon dioxide concentration will aggravate global warming,[3] causes a series of environmental problems such as sea level rise, abnormal climate, and land desertification.

\section{CCS technology}

At present, in order to ameliorate the growth of carbon dioxide concentration, carbon capture and storage (CCS) was proposed [4]. CCS allows carbon dioxide absorption and separation from industries to be transported to geological storage, rather than being emitted to the atmosphere. Although this option can effectively alleviate climate change via reducing the concentration of carbon dioxide in the atmosphere, not only a mass of capital needs to be invested, but there is also a risk of leak. Even in some countries and regions such as the United Kingdom and Brazil, their geological storage capacity is limited or in some cases only available offshore, the cost of transportation and storage is augmented [5]. A more serious economic obstacles is that CCS is an unprofitable investment which requires abundant input. Therefore, CCS can moderate global warming theoretically, but it cannot be applied until it overcomes economic and geological hurdles.

\section{CCU technology}

More importantly, as a massive, safe and renewable carbon resource, carbon dioxide can be converted into valuable materials and chemicals [6], make contributions to climate change [7]. In this context, a meaningful way, carbon capture and utilization (CCU), has attracted attention $[8,9]$. Compare to CCS, the biggest advantage of $\mathrm{CCU}$ is that the utilization of carbon dioxide is a profitable activity since the products can be sold. At the same time, CCU simplifies the subsequent procedure, storage of carbon dioxide which makes CCU cheaper and securer. Although carbon dioxide has high thermodynamic stability, it has great potential in fuel supply and organic synthesis, which promote us to explore brand new catalytic method of $\mathrm{CO} 2$ conversion.

\section{Constraints on $\mathrm{CO}_{2}$ conversion}

The biggest distinction between $\mathrm{CCU}$ and CCS is that CCU utilizes $\mathrm{CO} 2$ conversion production and benefits from it. Hence, $\mathrm{CO} 2$ conversion plays an important role in CCU. Although it possesses enormous potential, it is necessary to ameliorate existing synthetic technology due to the molecular properties and thermodynamic and kinetic constraints. The industrial utilizations of carbon

Corresponding author: andrew_xqn@163.com 
dioxide are mainly in synthesis urea, such as synthesis of salycilic acid, inorganic carbonate and synthesis of polycarbonates from epoxides [10]. The first three processes do not need catalyst because they are under mild thermodynamic and kinetic restraints, but they have been around for a century, which have limited prospect. More importantly, synthesis of polycarbonates from epoxides is subjected to high thermodynamic and kinetic restraints, but we can make it easier by using catalyst to lower the reaction's activation energy.

Based on this, synthesis of polycarbonates from epoxides has appealed to people. Therefore, how to explore catalytic system which makes $\mathrm{CO} 2$ conversion more efficient appears to be highly essential.

\section{Nanocatalysis}

\subsection{Introduction}

Catalytic technology is vital to energy and chemical industries. The production of chemicals and control of toxic gas discharge such as carbon monoxide and nitric oxide are all rely on catalytic technology. Catalysts are also important components of electrode since it can participate in reaction. In the field of solid phase catalyst, huge amounts of chemical absorption sites will accelerate the catalytic process. Therefore, increase of superficial area and decrease of particle diameter can markedly enhance the performance of catalyst [11].

Nano materials are widely utilized due to their small particle size, great proportion of surface particle and high mass surface area. In modern laboratories, active catalysts tend to be composed of well-designed nano particles.

\subsection{Application of gold nanoarrays in electrochemistry}

It is very common to use precious metals as catalyst in hydrogenation in cell. However, in these reactions, produce of hydrogen will be inevitable, result in a big security issue. After repeated research and practice, it has been found that gold can avoid this issue which also has great stability and efficiency.

Xiaokun $\mathrm{Ma}$ and his team did a research about catalytic performance of gold coated nickel nanoarrays in 2017. They firstly used template-assisted electrodeposition to obtain Ni nanowire arrays (NAs), and then utilized galvanostatic deposition of $\mathrm{Au}$ onto the Ni NAs without any conductive agents and binders to gain Au@Ni nanoarrays electrode. After that, they tested the stability and electrochemical property of $\mathrm{Au} @ \mathrm{Ni}$ NAs by letting it catalyze $\mathrm{NaBH} 4$ electro-oxidation in basic solutions.

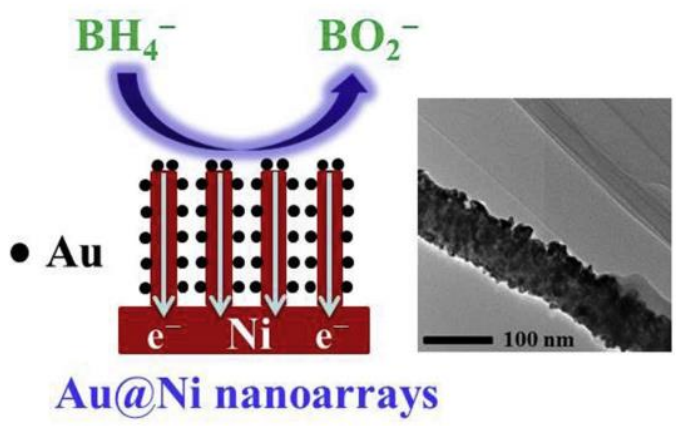

Fig. 1. Schematic diagram of catalytic oxidation of $\mathrm{BH}_{4}$ and surface structure characteristics of gold-nickel nanoarrays

This team performed SEM tests on Ni NAs and $\mathrm{Au} @ \mathrm{Ni} \mathrm{NAs}$ in order to study the situation of $\mathrm{Au}$ deposition and results are showed in figure 1.2. We can see that the profiles of whole nanowire arrays appeared to be distinctly rough which means Au densely coated on the Ni NAs surface. Otherwise, the deposition of $\mathrm{Au}$ offered great specific surface areas which guaranteed the great performance of $\mathrm{Au} @ \mathrm{Ni}$ NAs as catalyst.
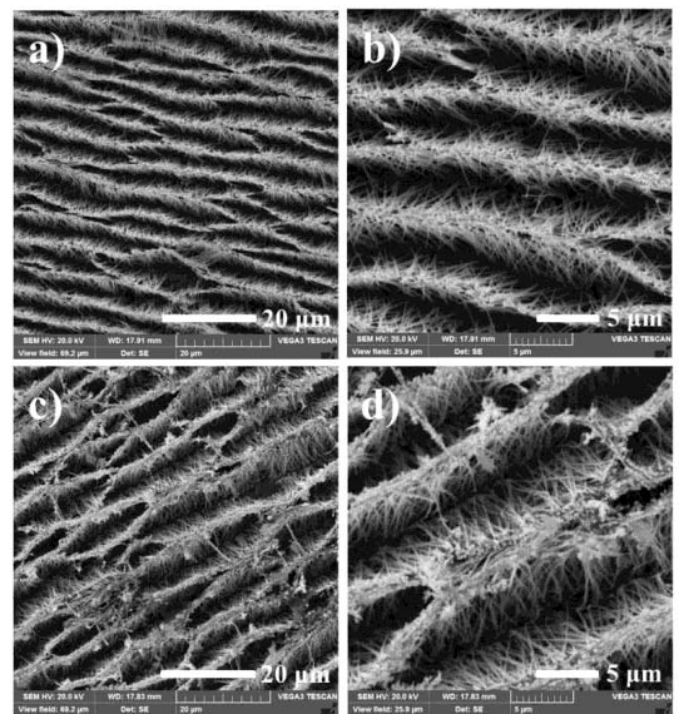

Fig. 2. SEM images of the Ni NAs (a and b) and Au@Ni NAs $(c$ and $d)$ electrodes under different magnification.

Then, they tested Ni NAs and Au@Ni NAs catalyze $\mathrm{NaBH} 4$ electro-oxidation acitivity in basic solution and drew cyclic voltammograms (CVs) picture. In figure 1.3a, they found that $\mathrm{Au} @ \mathrm{Ni}$ NAs showed similar redox responses to the gold catalysts in alkaline media which means gold formed on the Ni NAs. What's more, there was no obvious current response observed on Ni NAs for $\mathrm{NaBH} 4$ electro-oxidation in figure $1.3 \mathrm{~b}$, implying that the catalytic activity from the Ni NAs can be ignored and the high catalytic activity was resulted from the $\mathrm{Au}$ [12]. 

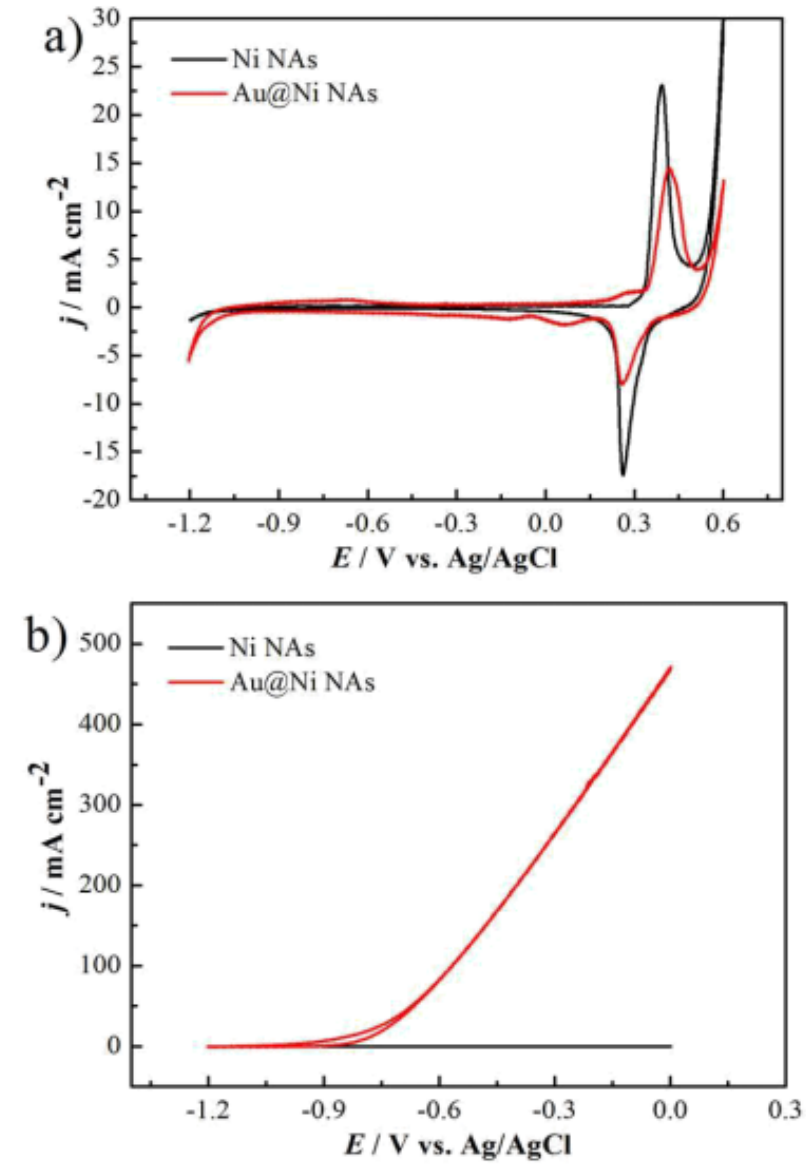

Fig. 3. CVs of the Ni NAs electrode and Au@Ni NAs electrode in $2.00 \mathrm{M} \mathrm{NaOH}(\mathrm{a})$; comparative CVs of the two electrodes in $2.00 \mathrm{M} \mathrm{NaOH}$ and $0.24 \mathrm{M} \mathrm{NaBH}_{4}$ (b); scanrate: $10 \mathrm{mV} \mathrm{s}^{-1}$.

The above research shows that nanoarray can provide large surface area and assures the high utilization of the catalyst. Meanwhile, it comes to a result that Au has high catalytic activity and stability. The $\mathrm{Au}$ nanoarray has great potential in electrochemistry and is hopefully applied in fuel cells.

\subsection{Current situation of $\mathrm{CO}_{2}$ conversion}

With the development of global energy economy and the popularization of chemical industries, people become more dependent on fossil fuel which raise carbon dioxide concentration in atmosphere. In order to alleviate this problem and accord with sustainable development to maintain steady economy growth, using renewable energy is accepted by majority of people. Among them, electrochemistry which use renewable electricity as an input to convert carbon dioxide into fuel and industrial product is widely applied. Nevertheless, this process need catalyst while there is only few meet criteria. Hence, survey of catalytic property and innovation is worthy.

Kendra P. Kuhl and his team tested trends in the electrocatalytic conversion of $\mathrm{CO} 2$ on a broad group of seven transition metal surfaces. They used a custom electrochemical cell, to conduct electrolysis experiments in $0.1 \mathrm{M} \mathrm{KHCO} 3$ electrolyte.
They conducted potentiostatic electrolysis experiments over a range of potentials for each metal studied and the average current density was shown in figure 2.1. The result is that $\mathrm{Ni}, \mathrm{Pt}$ and $\mathrm{Fe}$ are poor $\mathrm{CO} 2$ conversion catalysts because they mostly produce hydrogen. But $\mathrm{Au}, \mathrm{Ag}$ and $\mathrm{Zn}$, exhibit a high current efficiency for $\mathrm{CO} 2$ conversion which means they are better catalysts.

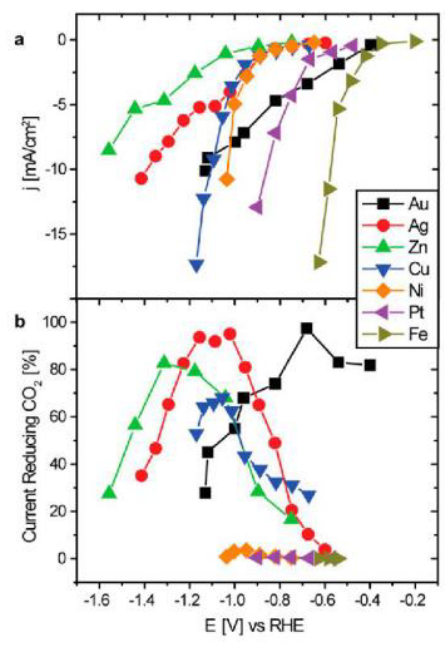

Fig. 4. (a) Current densities during $1 \mathrm{~h}$ long potentiostatic $\mathrm{CO}_{2}$ electrolysis experiments. (b) Current efficiencies (\%) for the $\mathrm{CO}_{2}$ reduction reaction $\left(\mathrm{CO}_{2} \mathrm{RR}\right)$ at each potential.

In order to gain deeper details to compare catalytic properties, they measured six of the seven metals (Pt, Ni, $\mathrm{Cu}, \mathrm{Au}, \mathrm{Ag}, \mathrm{Zn}$ ) at a common potential and plotted the partial current densities for the CO2RR at this potential versus CO binding energy which showed a volcanoshaped trend picture. As we can see, Au is at the peak of the plot which means it has the highest partial current density for the CO2RR and other metals with activity decreasing on either side. This represents that Au has the best potential as a catalyst among these transition metals [13].

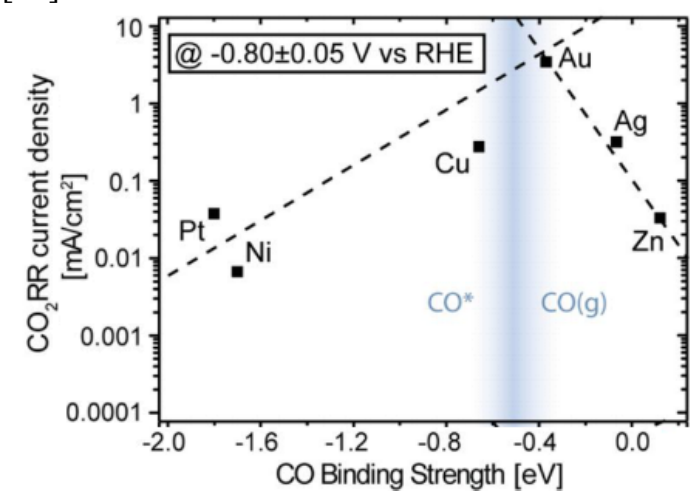

Fig. 5. Volcano plot of partial current density for $\mathrm{CO}_{2} \mathrm{RR}$ at $0.8 \mathrm{~V}$ vs $\mathrm{CO}$ binding strength.

\subsection{Au nanoarray catalyzes $\mathrm{CO}_{2}$ conversion}


After the above discussion, we expect to utilize $\mathrm{Au}$ nanoarray to catalyze the addition reaction of carbon dioxide and epoxides.

Usually, people utilize molecular self-assembly to automatically arrange themselves into nanoarray through a bottom-up approach. The self-assembly of nano particles is a delicate process which need ligand and cross-linking agent to provide repulsive force and spatial coherence to balance the gravitation between nano particles and control the spatial gap and particle size in arrays. Although there are numerous methods for producing nanoarray, Brust's is the most popular one in the field. In 1994, Brust and his team successfully prepared Au nanoparticles by utilizing two-phase system and redox reaction which can be summarized by two equations.

$\mathrm{AuCl}_{4}{ }^{-}(\mathrm{aq})+\mathrm{N}\left(\mathrm{C}_{8} \mathrm{H}_{17}\right)_{4}{ }^{+}\left(\mathrm{C}_{6} \mathrm{H}_{5} \mathrm{Me}\right)^{-} \rightarrow \mathrm{N}\left(\mathrm{C}_{8} \mathrm{H}_{17}\right)_{4}{ }^{+} \mathrm{AUC1} 1_{4}$ (C6H5Me)

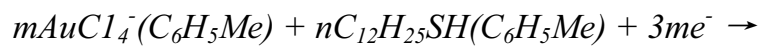

$4 m \mathrm{Cl}^{-}(\mathrm{aq})+\left(A u_{m}\right)\left(\mathrm{C}_{12} \mathrm{H}_{25} \mathrm{SH}\right) n\left(\mathrm{C}_{6} \mathrm{H}_{5} \mathrm{Me}\right)$ (2)

Firstly, they used tetraoctylammonium bromide as hase-transfer reagent to transfer $\mathrm{Au}$ from aqueous solution to toluene, and then reduced with aqueous sodium orohydride in the presence of dodecanethiol. What's more, an extraordinary property of these nanoparticles produced by the above process is that they can be tested and characterized as simple chemical compound. Brust et al. thought that this preparation technique not only provided a simple way for the synthesis of metallic clusters, but also could easily control cluster size by using different reaction conditions. All these advantages have attracted considerable attention, its following variation also shew up and achieve great success [14].

Zhao et al. summarized the state of synthesis in gold nanoparticle in 2013. It is mentioned that the combination of $\mathrm{Au}$ and thiol ligands to get stabilized gold nanoparticle is the energy favorite way. During the growth reaction of thiol and $\mathrm{Au}$, the $\mathrm{H}$ atom of thiol is lost and the $\mathrm{Au}-\mathrm{H}$ bonds has fluxional properties which makes it possible for $\mathrm{H}$ atoms to move quickly until two $\mathrm{Au}-\mathrm{H}$ bonds link together. In view of this advantage, Brust method has been widely applied and functionalized in thiolate-liganded AuNPs [15].

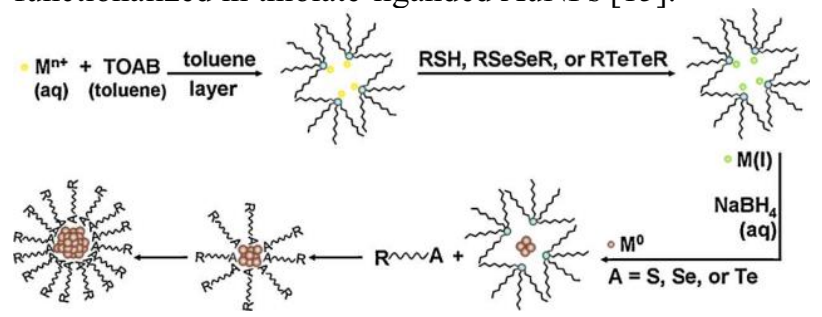

Fig. 6. Mechanism for chalcogenate-protected metal NP synthesis by the Brust-Schiffrin method.

Traditionally, people use flexible ligand and crosslinking agent because it is conducive to form twodimensional structure when they combine with chemicals by strong binding effect. Although flexible ligand and cross-linking agent with long chains are able to subtly stabilize nano particles location in arrays, the flexible chains tightly wrap central nano particles. In this way, the reduction of catalytic sites and superficial area causes the drop of efficiency, which is also the disadvantage of flexible cross-linking method.

Therefore, scientists expect to exposure central nano particles through rigid cross-linking. According to previous research, it is found that Au has strong binding effect, and is able to form covalent bond by binding with sulphur which can stabilize two-dimensional structure. Meanwhile, the application of $\mathrm{Au}$ nanoarray in electrochemistry and $\mathrm{CO} 2$ conversion has proved that $\mathrm{Au}$ has high catalytic efficiency.

In this context, we believe that Au can combine with rigid cross-linking agent to obtain Au nanoarray through self-assembly process. Its catalytic performance can be tested by letting it catalyze the addition reaction of carbon dioxide and epoxides and it is believed that this catalyst will show a great performance.

\section{Conclusion}

The idea that utilize $\mathrm{Au}$ nanoarray to catalyze $\mathrm{CO}_{2}$ conversion will make contributions to alleviate global warming. Not only does it open up a new path for application of Au nanoarray and design of heterogeneous catalysts, but also provides a unique catalytic method for CCU technology.

\section{References}

1. Olah G A, Prakash G K S and Goeppert A. Anthropogenic chemical carbon cycle for a sustainable future[J]. J. Am. Chem. Soc., 13333 (2011): 12881 12898.

2. Muradov N Z. How to produce hydrogen from fossil fuels without $\mathrm{CO}_{2}$ emission[J]. Int. J. Hydrogen Energy, 183 (1993): 211 215.

3. Mitchell J F. The "greenhouse" effect and climate change. Reviews of Geophysics, 271 (1989) : 115139.

4. Gibbins J, Chalmers H. Carbon capture and storage[J]. Energy policy, 3612 (2008): 4317-4322.

5. Cuéllar-Franca R M, Azapagic A. Carbon capture, storage and utilisation technologies: A critical analysis and comparison of their life cycle environmental impacts. Journal of $\mathrm{CO} 2$ utilization, 9 (2015): 82-102.

6. Zhou H, Mu S, Ren B H, et al. Organocatalyzed carboxylative cyclization of propargylic amides with atmospheric $\mathrm{CO}_{2}$ towards oxazolidine-2, 4-diones. Green chemistry, 215 (2019): 991-994.

7. Mac Dowell N, Fennell P S, Shah N, et al. The role of $\mathrm{CO}_{2}$ capture and utilization in mitigating climate change. Nature Climate Change, 74 (2017): 243249. 
8. Khoo H H, Bu J, Wong R L, et al. Carbon capture and utilization: Preliminary life cycle $\mathrm{CO}_{2}$, energy, and cost results of potential mineral carbonation. Energy Procedia, 4 (2011): 2494-2501.

9. Woodall C M, McQueen N, Pilorgé H, et al. Utilization of mineral carbonation products: current state and potential. Greenhouse Gases: Science and Technology, (2019).

10. Aresta M, Tommasi I. Carbon dioxide utilisation in the chemical industry. Energy conversion and management, 38 (1997): S373-S378.

11. Grzelczak M, Pérez-Juste J, Mulvaney $P$, et al. Shape control in gold nanoparticle synthesis. Chemical Society Reviews, 379 (2008): 1783-1791.

12. Ma X, Ye K, Wang G, et al. Facile fabrication of gold coated nickel nanoarrays and its excellent catalytic performance towards sodium borohydride electro-oxidation. Applied Surface Science, 414 (2017): 353-360.

13. Kuhl K P, Hatsukade T, Cave E R, et al. Electrocatalytic conversion of carbon dioxide to methane and methanol on transition metal surfaces. Journal of the American Chemical Society, 13640 (2014): 14107-14113.

14. Brust M, Walker M, Bethell D, et al. Synthesis of thiol-derivatised gold nanoparticles in a two-phase liquid-liquid system. Journal of the Chemical Society, Chemical Communications, 7 (1994): 801802.

15. Zhao P, Li N, Astruc D. State of the art in gold nanoparticle synthesis. Coordination Chemistry Reviews, 257 3-4 (2013): 638-665. 